\title{
IKKß suppression of TSC1 function links the mTOR pathway with insulin resistance
}

\author{
DUNG-FANG LEE ${ }^{1,2}$, HSU-PING KUO ${ }^{1,2}$, CHUN-TE CHEN $^{1,2}$, YONGKUN WEI ${ }^{1}$, \\ CHAO-KAI CHOU ${ }^{1,2}$, JEN-YU HUNG ${ }^{1,4}$, CHIA-JUI YEN ${ }^{1,5}$ and MIEN-CHIE HUNG ${ }^{1,2,3}$ \\ ${ }^{1}$ Department of Molecular and Cellular Oncology, The University of Texas M.D. Anderson Cancer Center; \\ ${ }^{2}$ The University of Texas Graduate School of Biomedical Sciences at Houston, Houston, TX 77030, USA \\ ${ }^{3}$ Center for Molecular Medicine and Graduate Institute of Cancer Biology, China Medical \\ University and Hospital, Taichung, Taiwan
}

Received June 13, 2008; Accepted July 29, 2008

DOI: 10.3892/ijmm_00000065

\begin{abstract}
The proinflammatory cytokine TNF $\alpha$ is one of the factors that links obesity-derived chronic inflammation with insulin resistance. Activation of mTOR signaling pathway has been found to suppress insulin sensitivity through serine phosphorylation and the inhibition of IRS1 by mTOR and its downstream effector, S6K1. It remains elusive that whether the mTOR pathway has a role in TNF $\alpha$-mediated insulin resistance. In the present study, we demonstrated that $\mathrm{TNF}_{\alpha}-$ IKK $\beta$-mediated inactivation of TSC1 resulted in increasing phosphorylation of IRS1 serine 307 and serine 636/639, impaired insulin-induced glucose uptake, tyrosine phosphorylation of IRS1, and the association between IRS1 and PI3K p85. Furthermore, a higher expression of pIKK $\beta$ (S181), pTSC1(S511), and pS6(S240/244) was found in livers obtained from both C57BL/6J mice on a high-fat diet and B6.V-Lep ${ }^{\mathrm{ob} / \mathrm{J}}$ mice. Collectively, dysregulation of the TSC1/ TSC2/mTOR signaling pathway by IKK $\beta$ is a common molecular switch for both cancer pathogenesis and diet- and obesity-induced insulin resistance.
\end{abstract}

\section{Introduction}

Insulin action is essential for growth, development, and metabolism. Upon stimulation with insulin, insulin receptor (IR) stimulates its intrinsic tyrosine kinase activity and phos-

Correspondence to: Dr Mien-Chie Hung, The University of Texas M.D. Anderson Cancer Center, Department of Molecular and Cellular Oncology, Unit 108, 1515 Holcombe Blvd., Houston, TX 77030, USA

E-mail: mhung@mdanderson.org

Present addresses: ${ }^{4}$ Department of Internal Medicine, Kaohsiung Medical University Hospital, Kaohsiung 807, ${ }^{5}$ Department of Medicine, National Chen Kung University, Tainan 70428, Taiwan, R.O.C.

Key words: ІкB kinase $\beta$, tuberous sclerosis 1 , mammalian target of rapamycin pathway, insulin resistance phorylates IR substrate 1 (IRS1). The tyrosine-phosphorylated IRS1 functions as an adaptor to activate downstream phosphoinositide 3-kinase (PI3K)/AKT signaling, which leads to enhancement of glucose uptake, synthesis of protein and glycogen, and execution of the growth-promoting and metabolic effects of insulin. In contrast with the positive effect of tyrosine phosphorylation of IRS1, serine phosphorylation actually inhibits the function of IRS1 (1). Although serine phosphorylation of IRS1 induced by insulin can function as a feedback control, other factors, such as proinflammatory cytokines, also increase phosphorylation of IRS1 and function as negative regulators. A large number of kinases function as IRS1 serine kinases and play negative regulatory roles in insulin action. These include mammalian target of rapamycin (mTOR)-mediated phosphorylation of IRS1 serine 636 (Ser636) and serine 639 (Ser639) (2), ribosomal S6 kinase 1 (S6K1)-mediated phosphorylation of IRS1 serine 307 [Ser307 (mouse serine 302) (Ser302)] (3) and serine 1101 (4), IкB kinase $\beta$ (IKKß)- and c-Jun N-terminal kinase (JNK)-mediated phosphorylation of IRS 1 serine 312 [Ser312 (mouse Ser307)] $(5,6)$, and protein kinase $\zeta$-mediated phosphorylation of IRS1 serine 323 (mouse serine 318) (7).

Increased serine phosphorylation of IRS1 leads to insulin resistance, which is commonly associated with obesity. A growing body of evidence suggests that obesity-derived chronic inflammation is a key factor for obesity-mediated insulin resistance. Of the proinflammatory cytokines involved in obesity-mediated insulin resistance, tumor necrosis factor $\alpha$ $(\mathrm{TNF} \alpha)$ is considered a major mediator that contributes to this resistance. Elevated plasma $\mathrm{TNF} \alpha$ levels are positively correlated with obesity and insulin resistance in obese humans and animals (Zucker fa/fa rats, ob/ob mice, and $\mathrm{db} / \mathrm{db}$ mice) $(8,9)$ and are significantly related to the development of type 2 diabetes. Researchers demonstrated that the depletion of $\mathrm{TNF} \alpha$ by a soluble TNF $\alpha$ receptor improved insulin action in a murine model of obesity (9), indicating the vital role of TNF $\alpha$ signaling in obesity-mediated insulin resistance.

Investigators have proposed that activation of two serine kinases, IKK 3 and JNK, is involved in TNF $\alpha$-induced insulin resistance. IKKß- and JNK-mediated phosphorylation of IRS1 Ser312 directly inhibits IR-induced tyrosine phos- 
phorylation of IRS1 and disrupts the association between IRS1 and p85 (a PI3K regulatory subunit), thereby interfering with insulin signaling. Although the involvement of IKK 3 and $\mathrm{JNK}$ in $\mathrm{TNF} \alpha$-mediated insulin resistance is generally approved, recent studies raise interesting possibilities that other mechanisms may be involved in this inhibition. For instance, activation of the mTOR pathway suppresses insulin signaling by modulating the serine phosphorylation of IRS 1 , which serves as a feedback regulator of the insulin signaling pathway. mTOR and its downstream effector S6K1 suppress IRS1 activity by directly phosphorylating IRS1 at Ser636/ Ser639 and Ser307, respectively, which leads to desensitization of insulin signaling $(3,10)$. Remarkably, S6k1-deficient mice are protected against nutritionally and genetically driven insulin resistance (11), and genetic loss of either tuberous sclerosis 1 (TSC1) or tuberous sclerosis 2 (TSC2) results in insulin resistance by increasing the phosphorylation of IRS1 at Ser302 (3), suggesting that hyper-activation of the mTOR signaling cascade attenuates IR signaling.

Furthermore, we recently found that $\mathrm{TNF} \alpha$ activates the mTOR pathway through IKKß-mediated phosphorylation and inactivation of TSC1, which contributes to inflammationmediated tumorigenesis via increased vascular endothelial growth factor production $(12,13)$. Collectively, this raises the question of whether IKKß-induced mTOR activation contributes to $\mathrm{TNF} \alpha$-mediated insulin resistance. In the present study, we examined the role of phosphorylation of TSC 1 by IKK $\beta$ in $\mathrm{TNF} \alpha$-induced insulin resistance, and uncovered a previously unrecognized signaling mechanism distinct from the established model by which $\mathrm{TNF} \alpha$ induces insulin resistance through IKKß- and JNK-mediated phosphorylation of IRS1 Ser312. Based on our present and previous findings (12), we concluded that dysregulation of the TSC1/TSC2/mTOR signaling pathway by IKK 3 is a common molecular switch for both cancer pathogenesis and diet- and obesity-induced insulin resistance. Our finding may provide a target for clinical intervention for treatment of dietand obesity-induced insulin resistance.

\section{Materials and methods}

Antibodies and chemicals. We used antibodies against Myc (11667203001; Roche), S6K1 (SC-230); IRS1 (SC-559); I $\mathrm{B} \alpha$ (SC-371) (the above 3 from Santa Cruz Biotechnology), phosphorylated S6K1(T389) [pS6K1(T389), 9205]; phosphorylated 4EBP1(S65) [p4EBP1(S65), 9451]; 4EBP1 (9452); phosphorylated S6(S240/244) [pS6(S240/244)], 2215; phosphorylated IRS 1(S307) \{phosphorylated IRS1(S302) [pIRS1(S302) in mice], 2384\}; phosphorylated IRS1(S636/639) [pIRS1(S636/639), 2388]; phosphorylated IKKß(S181) [pIKKß(S181), 2681]; phosphorylated AKT(S473) [pAKT(S473), 9271 and 9277] (the above 8 from Cell Signaling Technology), PI3K p85 (06-195); phosphorylated Tyr 4G10 (05-321) (the above 2 from Upstate Biotechnology), and actin (A2066; Sigma). Antibodies against TSC1 Ser511 phosphorylation were described previously (12). Recombinant human TNF $\alpha$ was purchased from Roche.

Stable transfectants. MDA-MB-453 TSC1 stable transfectants were selected and maintained in Dulbecco's modified Eagle's
medium/F12 medium supplemented with $10 \%$ fetal bovine serum and $500 \mu \mathrm{g} / \mathrm{ml} \mathrm{G} 418$.

Immunoprecipitation and immunoblotting. Cells were lysed in RIPA-B buffer (20 mM Na $2 \mathrm{HPO}_{4}$, pH 7.4, $150 \mathrm{mM} \mathrm{NaCl}$, $1 \%$ Triton $\mathrm{X}-100)$ with protease inhibitors (1 $\mathrm{mM}$ phenylmethylsulfonyl fluoride, $5 \mathrm{mM} \mathrm{NaF}, 2 \mathrm{mM}$ sodium orthovanadate, $3 \mu \mathrm{g} / \mathrm{ml}$ aprotinin, and $750 \mu \mathrm{g} / \mathrm{ml}$ benzamidine) and processed as described previously (14). For immunoprecipitation, samples were precleaned with protein A-agarose for $1 \mathrm{~h}$ at $4^{\circ} \mathrm{C}$. Precleaned cell lysates were immunoprecipitated with an anti-IRS1 antibody overnight at $4^{\circ} \mathrm{C}$, incubated with protein A-agarose for $3 \mathrm{~h}$ at $4^{\circ} \mathrm{C}$, washed with ice-cold RIPA buffer containing protease inhibitors four times, resolved using sodium dodecyl sulfate (SDS)-polyacrylamide gel electrophoresis (PAGE), and transferred to polyvinylidene difluoride membranes. For immunoblotting, membranes were blocked with TBST buffer (10 mM Tris- $\mathrm{HCl}, \mathrm{pH} 7.9,150 \mathrm{mM} \mathrm{NaCl}$, $0.05 \%$ Tween 20 ) with either $5 \%$ bovine serum albumin or $5 \%$ skim milk, incubated with indicated primary antibodies, subsequently incubated with horseradish peroxidase-conjugated secondary antibodies, and detected using enhanced chemiluminescence (Amersham Biosciences).

Glucose uptake assay. An insulin-stimulated glucose uptake assay was performed for detecting insulin response, as described previously (15). Briefly, MDA-MB-453 TSC1 stable transfectants plated in a six-well plate with complete medium (Dulbecco's modified Eagle's medium/F12 medium containing $10 \%$ fetal calf serum) were washed with phosphatebuffered saline (PBS) and serum-starved overnight. Cells were either left untreated or treated with $10 \mu \mathrm{M}$ insulin for 30 min followed by the addition of 2-deoxy-D-[2,6- $\left.{ }^{3} \mathrm{H}\right]$ glucose $(1 \mu \mathrm{Ci} / \mathrm{ml})$ and 2 -deoxy-D-glucose $(0.1 \mathrm{mM})$ for an additional $5 \mathrm{~min}$ at $37^{\circ} \mathrm{C}$. After washing three times with icecold PBS, cells were solubilized in $0.4 \mathrm{ml}$ of $1 \% \mathrm{SDS}$, and the level of tritium radioactivity was determined using liquid scintillation counting. Nonspecific uptake was measured in the presence of cytochalasin $\mathrm{B}(20 \mu \mathrm{M})$ and subtracted from the total uptake to determine the specific level of glucose uptake.

Animal model of insulin resistance. Male 6-week-old C57BL/6J and B6.V-Lep ${ }^{\mathrm{ob} / \mathrm{J}}$ (ob/ob) mice were purchased from The Jackson Laboratory and maintained in the animal care facility of the Department of Veterinary Medicine at The University of Texas M.D. Anderson Cancer Center. The C57BL/6J mice were fed either a high-fat diet (HFD) (D12331; Research Diets) or a normal chow diet (NCD), whereas the ob/ob mice were fed an NCD only. Diet-induced insulin resistance was assessed in the mice as described previously (16). After 16 weeks of feeding on these diets, the mice were sacrificed, and frozen sections of their livers were prepared for immunofluorescent staining. All animal studies were performed following an animal protocol approved by the Institutional Animal Care and Use Committee at M.D. Anderson Cancer Center.

Immunofluorescent staining. Immunofluorescent staining of the frozen liver sections was performed as described previously (17). Briefly, 4- $\mu \mathrm{m}$ frozen sections were fixed; washed with 
A

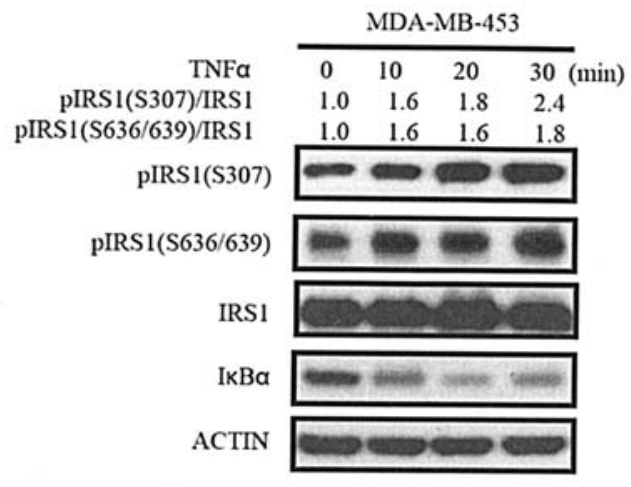

B

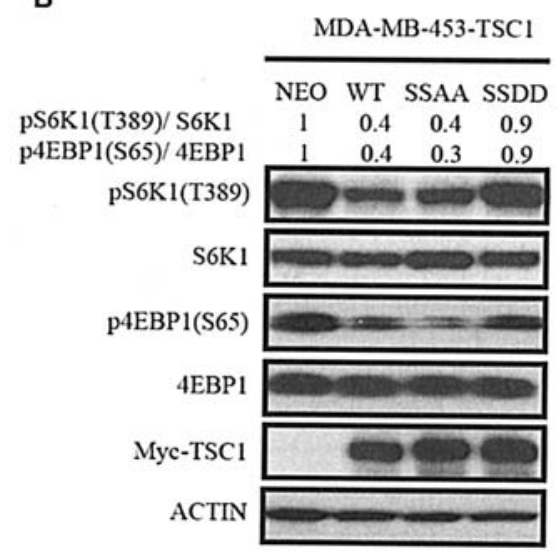

C

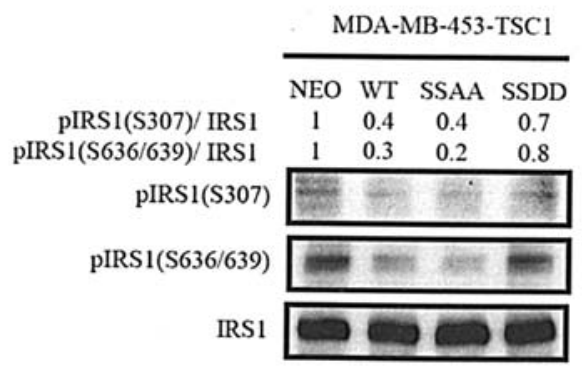

Figure 1. TNF $\alpha$-mediated insulin resistance via inappropriate activation of the TSC1/mTOR/S6K1 signaling cascade. A) Phosphorylation of IRS1 Ser307 and Ser636/639 was induced by TNF $\alpha$ in MDA-MB-453 cells. Cells were serum-starved overnight and then treated with $20 \mathrm{ng} / \mathrm{ml} \mathrm{TNF} \alpha$ in a timecourse study. Cell extracts were prepared and resolved using SDS-PAGE and immunoblotted with anti-pIRS1(S307), anti-pIRS1(S636/639), antiIRS1, anti-IкB $\alpha$, and anti-ACTIN antibodies. B) Endogenous expression levels of pS6K(T389) and p4EBP1(S65) were measured in MDA-MB-453 cells stably transfected with various TSC1 mutants or a vector control (NEO). MDA-MB-453-TSC1 stable transfectants were selected based on the equal amount of TSC1 expression in the transfectants. Transfectants were serum-starved overnight and lysed with RIPA-B buffer with protease inhibitors. Extracts of stable transfectants were resolved using SDS-PAGE and immunoblotted with anti-pS6K1(T389), anti-S6K1, anti-p4EBP1(S65), anti-4EBP1, anti-Myc, and anti-ACTIN antibodies. C) The expression levels of pIRS1(S307) and pIRS1(S636/639) were higher in MDA-MB-453TSC1(SSDD) than in MDA-MB-453-TSC1(SSAA) stable transfectants. MDA-MB-453-TSC1 stable transfectants were serum-starved overnight and lysed with RIPA-B buffer with protease inhibitors. Extracts of stable transfectants were resolved using SDS-PAGE and immunoblotted with antipIRS1(S307), anti-pIRS1(S636/639), and anti-IRS1 antibodies.

PBS; incubated with antibodies against pIKKß(S181), pTSC1(S511), pS6(S240/244), and pAKT(S473); incubated with goat anti-rabbit immunoglobulin $\mathrm{G}$ conjugated with
A
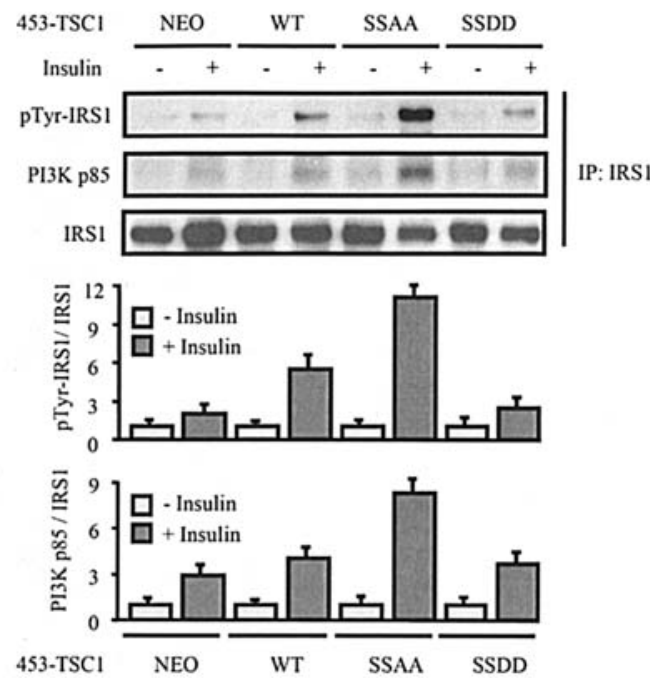

B

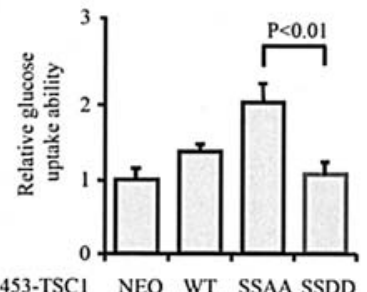

Figure 2. IKKB-mediated TSC1 phosphorylation impairs insulin action. A) Insulin-induced tyrosine phosphorylation of IRS1 and the association between IRS1 and PI3K p85 were significantly higher in MDA-MB-453TSC1(SSAA) than in MDA-MB-453-TSC1(SSDD) stable transfectants. MDA-MB-453-TSC1 stable transfectants were serum-starved overnight and then treated with $10 \mu \mathrm{M}$ insulin for $30 \mathrm{~min}$. Cell extracts were prepared and immunoprecipitated with an anti-IRS1 antibody. The immunoprecipitated extracts were resolved using SDS-PAGE and immunoblotted with anti-pTyr, anti-p85, and anti-IRS1 antibodies. The experiment was quantified to yield the relative ratio of pTyr-IRS1 or PI3K p85 to total IRS1. The plots represent the mean of three independent experiments, and the error bars represent SD B) Insulin-induced glucose uptake was higher in MDA-MB-453TSC1(SSAA) than in MDA-MB-453-TSC1(SSDD) stable transfectants 453-TSC1 stable transfectants were serum-starved overnight and treated with $10 \mu \mathrm{M}$ insulin for $30 \mathrm{~min}$ followed by the addition of 2-deoxy-D-[2,6$\left.{ }^{3} \mathrm{H}\right]$ glucose $(1 \mu \mathrm{Ci} / \mathrm{ml})$ and 2-deoxy-D-glucose $(0.1 \mathrm{mM})$ for an additional $5 \mathrm{~min}$ at $37^{\circ} \mathrm{C}$. PBS-washed transfectants were solubilized in $0.4 \mathrm{ml}$ of $1 \%$ SDS and the level of tritium radioactivity was measured using liquid scintillation counting. Nonspecific uptake was measured in the presence of cytochalasin B $(20 \mu \mathrm{M})$ and subtracted from the total uptake to determine the specific glucose uptake level. The relative glucose uptake ability in response to insulin is shown as the average of three independent experiments. The Student's t-test was used for statistical analysis.

fluorescein isothiocyanate in the dark; and examined under a fluorescent microscope (Zeiss). The nuclei of liver cells were stained with 4,6-diamidino-2-phenylindole.

\section{Results}

TNF $\alpha$-mediated insulin resistance via inappropriate activation of the TSC1/mTOR/S6K1 signaling cascade. Activation of the mTOR pathway functions as a feedback regulator of insulin action by increasing phosphorylation of IRS1 at Ser307 [pIRS(S307)] and Ser636/639 [pIRS (S636/639)] by S6K1 


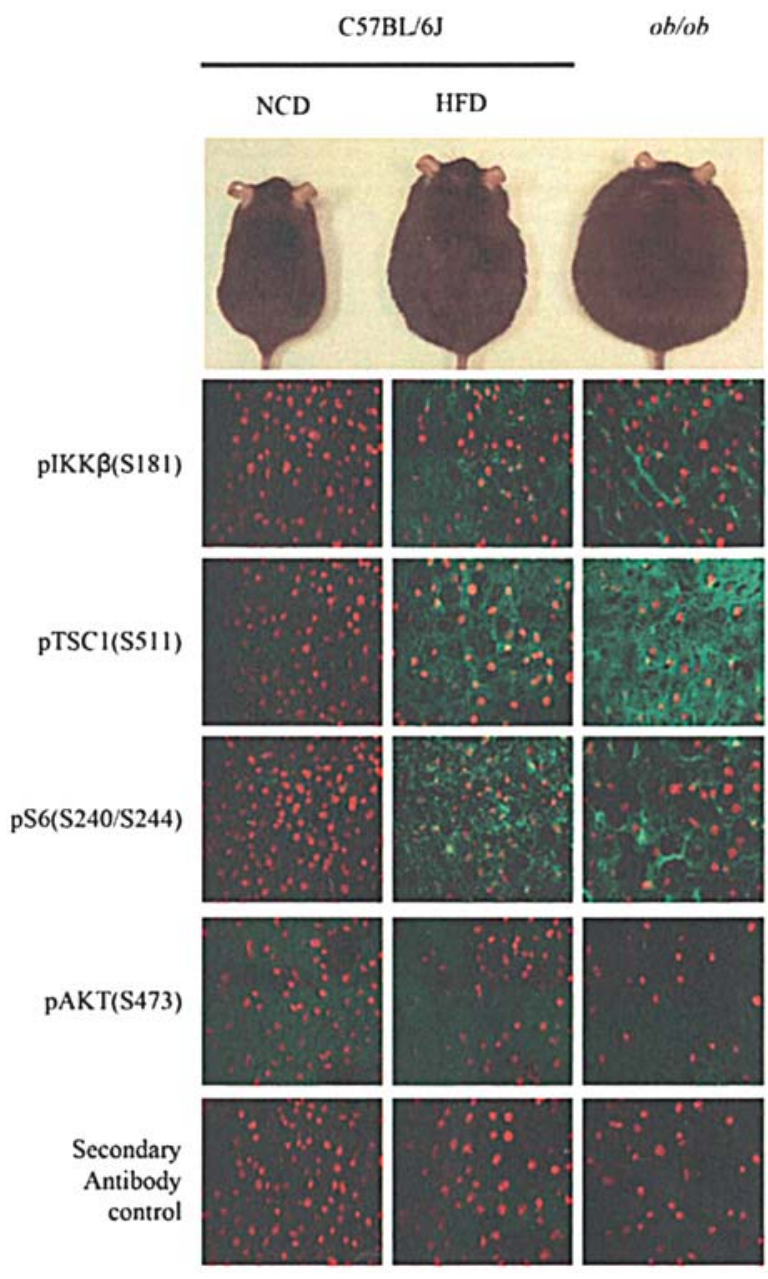

Figure 3. Up-regulation of IKKß/TSC1/mTOR/S6K1 signaling in C57BL/6J mice on an HFD and ob/ob mice. An immunostaining assay was performed to determine the $\mathrm{pIKK} \beta(\mathrm{S} 181)$, pTSC1(S511), pS6(S240/244), and pAKT(S473) status in livers obtained from the study mice. Male wild-type C57BL/6J mice were fed either an HFD or an NCD, whereas the ob/ob mice were fed an NCD only. After 16 weeks, the mice were sacrificed, and frozen liver sections obtained from them were fixed; stained with anti-pIKKB (S181), anti-pTSC1(S511), anti-pS6(S240/244), or anti-pAKT(S473) antibodies (green); and counterstained with TOPRO-3 dye (red) to visualize the nuclei of liver cells

(3) and mTOR (2), respectively. Also, studies showed that treatment with rapamycin prevents the development of insulin resistance by inhibiting the serine phosphorylation of IRS1 $(3,10)$. Recently, we demonstrated that the pro-inflammatory cytokine $\mathrm{TNF} \alpha$ activates the mTOR pathway and results in tumor angiogenesis $(12,13)$. This raised the intriguing question of whether activation of the mTOR pathway contributes to $\mathrm{TNF} \alpha$-induced insulin resistance by increasing phosphorylation of IRS1 Ser307 and Ser636/639. In the present study, we found that treatment with $\mathrm{TNF} \alpha$ substantially induced the expression of pIRS1(S307) and pIRS1(S636/639) in MDA-MB-453 cells within 10 to $30 \mathrm{~min}$ (Fig. 1A). To further address this issue, we used stable transfectants of the paired stable transfectants MDA-MB453-TSC1(SSAA) and MDA-MB-453-TSC1(SSDD), which have constitutive suppression and activation, respectively, of the IKKß/TSC1/mTOR pathway, to determine whether suppression of TSC1 function by IKK $\beta$ has a role in $\mathrm{TNF} \alpha$ mediated insulin resistance. In determining the phosphorylation

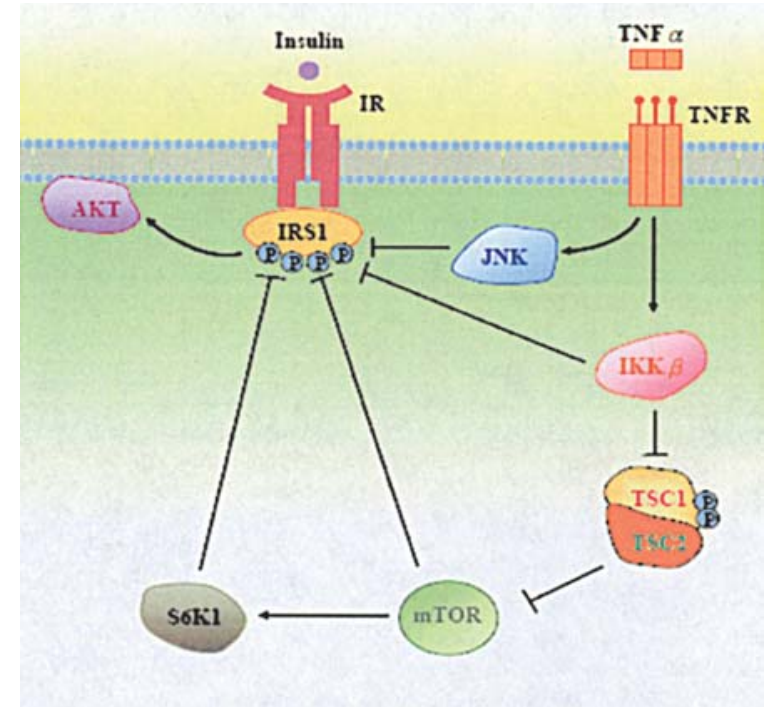

Figure 4. A model of the involvement of TNF $\alpha$-mediated signaling pathways in insulin resistance. Multiple IRS1 serine kinases, including IKK $\beta$, JNK, mTOR, and S6K1, are involved in TNF $\alpha$-induced serine phosphorylation and inactivation of IRS1, resulting in interference with insulin action.

status of S6K1 at T389 [pS6K1(T389)] and 4EBP1 at S65 [p4EBP1(S65)], which are two downstream phosphorylation sites of mTOR, we found higher expression of pS6K1(T389) and $\mathrm{p} 4 \mathrm{EBP} 1$ (S65) in 453-TSC1(SSDD) stable transfectants than in MDA-MB-453-TSC1(WT) or MDA-MB-453TSC1(SSAA) stable transfectants (Fig. 1B), confirming our previous finding that IKK 3 phosphorylation of TSC1 activates the mTOR pathway $(12,13)$. This result prompted us to examine whether IKKß-derived phosphorylation of TSC1 can serve as a switch for TNF $\alpha$ - and mTOR-induced phosphorylation of IRS1 at Ser307 and Ser636/639. Examination of the serine phosphorylation status of IRS1 [pIRS(S307) and pIRS(S636/639)] in various MDA-MB-453-TSC1 stable transfectants showed higher expression of pIRS(S307) and pIRS(S636/639) in MDA-MB-453-TSC1(SSDD) stable transfectants than in MDA-MB-453-TSC1(WT) or MDA-MB453-TSC1(SSAA) stable transfectants (Fig. 1C), indicating that activation of mTOR signaling by IKKß-mediated phosphorylation of TSC1 increases phosphorylation of IRS1 at Ser307 and Ser636/639 through up-regulation of S6K1 and mTOR activity, respectively.

IKKß-mediated TSC1 phosphorylation impairs insulin action. Since serine phosphorylation of IRS1 has a significant role in counteracting insulin action (IR-induced IRS 1 tyrosine phosphorylation and association with PI3K p85) $(1,18,19)$, increased serine phosphorylation of IRS1 by IKKß/TSC1/ mTOR signaling likely inhibits insulin response. To further validate the physiological relevance of our observations described above, we studied the effect of IKKß-mediated phosphorylation of TSC1 on insulin-induced tyrosine phosphorylation of IRS1, the association between IRS1 and PI3K p85, and the level of glucose uptake by insulin stimulation. As expected, we observed less of a response to insulininduced tyrosine phosphorylation of IRS, less of an association between IRS1 and PI3K p85 (Fig. 2A), and a lower level of glucose uptake (Fig. 2B) in MDA-MB-453-TSC1(SSDD) 
stable transfectant than in MDA-MB-453-TSC1(WT) or MDA-MB-453-TSC1(SSAA) stable transfectants. Thus, activation of the mTOR/S6K1 signaling pathway by IKKßinduced phosphorylation and inactivation of TSC1 may contribute to $\mathrm{TNF} \alpha$-mediated insulin resistance by increasing serine phosphorylation of IRS1.

Up-regulation of $I K K \beta / T S C 1 / m T O R / S 6 K 1$ signaling in $C 57 B L / 6 J$ mice on an HFD, and in ob/ob mice. Obesity is significantly associated with insulin resistance and is a state of chronic inflammation as indicated by increased plasma concentrations of TNF $\alpha$ in obese humans and animals $(8,9)$. Protection against obesity-induced insulin resistance by either knockout or neutralization of TNF $\alpha$ by a soluble TNF $\alpha$ receptor provides solid evidence supporting the concept that $\mathrm{TNF} \alpha$ has an essential role in obesity-mediated insulin resistance (9). To further determine whether suppression of TSC1 function by $\mathrm{TNF} \alpha$-activated IKK $\beta$ is related to obesityinduced insulin resistance, we sought to determine whether the $\mathrm{TNF} \alpha / \mathrm{IKK} \beta / \mathrm{TSC} 1 / \mathrm{mTOR} / \mathrm{S} 6 \mathrm{~K} 1$ pathway is activated in mice on an HFD and in genetically obese mice. We measured the $\mathrm{pIKK} ß(\mathrm{~S} 181)$, pTSC1(S511), and pS6(S240/244) (which is phosphorylated by $\mathrm{S} 6 \mathrm{~K} 1$ and used as an indicator of S6K1 activity) status in frozen section of livers obtained from C57BL/6J mice that were fed the HFD and genetically obese $\mathrm{C} 57 \mathrm{BL} / 6 \mathrm{~J} \mathrm{ob} / \mathrm{ob}$ mice, in which leptin deficiency leads to hyperglycemia, hyperinsulinemia, and insulin resistance (20). We found that the expression of pIKKß(S181), pTSC1(S511), and pS6(S240/244) was significantly higher in livers obtained from 16-week-old HFD C57BL/6J mice and ob/ob mice than in those obtained from NCD C57BL/6J mice (Fig. 3). Since insulin resistance impairs the PI3K/AKT signaling pathway, we determined the pAKT(S473) status in these livers. We observed a lower expression of pAKT(S473) in the livers obtained from HFD C57BL/6J mice and C57BL/6J ob/ob mice than in those obtained from NCD C57BL/6J mice (Fig. 3). These observations indicated that up-regulation of mTOR and S6K1 activity via suppression of TSC1 by IKKß may contribute to diet- and obesity-induced insulin resistance.

\section{Discussion}

$\mathrm{TNF} \alpha$ has an important role in mediating diet- and obesityinduced insulin resistance by increasing serine phosphorylation of IRS1, which inhibits insulin action. Several studies have suggested that IKKß and JNK are central coordinators in the regulation of $\mathrm{TNF} \alpha$-induced insulin resistance $(16,21,22)$, whereas the cellular and molecular mechanisms, by which TNF $\alpha$ impairs IRS1 function, are not fully elucidated. The findings of the present study of dietand obesity-induced insulin resistance in murine models suggest that suppression of TSC1 by IKKß activates mTOR and S6K1, which in turn phosphorylate IRS1 at Ser636/639 and Ser307, thereby inhibiting IRS1 function). Based on these and previous findings, herein we propose a model in which the high levels of $\mathrm{TNF} \alpha$ secreted by adipocytes and infiltrating macrophages (23) decrease the insulin response of cells to insulin through IKKß-, JNK-, mTOR-, and S6K1mediated phosphorylation and inactivation of IRS 1 in obese populations (Fig. 4). This in turn prevents glucose uptake by
GLUT4 and increases glucose concentrations in the blood, symptoms of diabetes. In addition to TNF $\alpha$, interleukin-6 (IL-6) is a pro-inflammatory cytokine involved in obesity-derived insulin resistance via the signal transducer and activator of transcription 3/suppressor of cytokine signaling 3 (STAT3) pathway. Notably, HFD- and obesity-derived increases in IL-6 expression may also occur due to transcriptional up-regulation by $\mathrm{TNF} \alpha / \mathrm{IKK} \beta$-induced nuclear factor $\kappa \mathrm{B}(\mathrm{NF}-\kappa \mathrm{B})$ activation (22). Collectively, these findings emphasize the vital role of $\mathrm{TNF} \alpha / \mathrm{IKK} \beta$ signaling in diet- and obesity-induced insulin resistance.

Importantly, dysregulation of TNF $\alpha$ contributes not only to obesity-mediated insulin resistance but also to cancer development. We recently demonstrated that the upregulation of the IKK $3 / \mathrm{TSC} 1 / \mathrm{mTOR} / \mathrm{S} 6 \mathrm{~K} 1$ signaling pathway enhances angiogenesis and culminates in breast cancer development. Clinical studies further suggest that dysregulation of this pathway is associated with poor clinical outcome of breast cancer. Findings of our present study and previous work $(12,13)$ suggest that the interaction between IKKß and TSC1 is a molecular switch for triggering both cancer and obesitymediated type 2 diabetes and offers a rationale for the role of obesity as a risk factor for both diseases (24).

\section{Acknowledgements}

We would like to thank Don Norwood for editing the manuscript. This work was partially supported by National Institutes of Health, grants R01 CA109311, P01 CA099031; Kadoorie Charitable Foundations; The National Breast Cancer Foundation, Inc; and a Breast Cancer Research Foundation grant, to M.-C.H; a predoctoral fellowship from the USA Army Breast Cancer Research Program (grant W81XWH-051-0252) and the T.C. Hsu Endowed Memorial, Andrew Sowell-Wade Huggins, Presidents' Research Scholarships, and Alfred Knudson Jr. Outstanding Dissertation Award from The University of Texas Graduate School of Biomedical Sciences at Houston, to D.-F.L; a predoctoral fellowship from the USA Army Breast Cancer Research Program (grant W81XWH-08-1-0397) and the Andrew Sowell-Wade Huggins Scholarship from The University of Texas Graduate School of Biomedical Sciences at Houston, to H.-P.K. and a predoctoral fellowship from the USA Army Breast Cancer Research Program (grant W81XWH-06-10709) to C.-K.C.

\section{References}

1. Zick Y: Ser/Thr phosphorylation of IRS proteins: a molecular basis for insulin resistance. Sci STKE 2005: pe4, 2005.

2. Ozes ON, Akca H, Mayo LD, et al: A phosphatidylinositol 3kinase/Akt/mTOR pathway mediates and PTEN antagonizes tumor necrosis factor inhibition of insulin signaling through insulin receptor substrate-1. Proc Natl Acad Sci USA 98: 4640-4645, 2001.

3. Harrington LS, Findlay GM, Gray A, et al: The TSC1-2 tumor suppressor controls insulin-PI3K signaling via regulation of IRS proteins. J Cell Biol 166: 213-223, 2004.

4. Tremblay F, Brule S, Hee Um S, et al: Identification of IRS-1 Ser-1101 as a target of S6K1 in nutrient- and obesity-induced insulin resistance. Proc Natl Acad Sci USA 104: 14056-14061, 2007.

5. Gao Z, Hwang D, Bataille F, et al: Serine phosphorylation of insulin receptor substrate 1 by inhibitor kappa B kinase complex. J Biol Chem 277: 48115-48121, 2002. 
6. Aguirre V, Uchida T, Yenush L, Davis R and White MF: The c-Jun $\mathrm{NH}(2)$-terminal kinase promotes insulin resistance during association with insulin receptor substrate- 1 and phosphorylation of Ser(307). J Biol Chem 275: 9047-9054, 2000.

7. Moeschel K, Beck A, Weigert C, et al: Protein kinase C-zetainduced phosphorylation of Ser318 in insulin receptor substrate-1 (IRS-1) attenuates the interaction with the insulin receptor and the tyrosine phosphorylation of IRS-1. J Biol Chem 279: 25157-25163, 2004.

8. Mantzoros CS, Moschos S, Avramopoulos I, et al: Leptin concentrations in relation to body mass index and the tumor necrosis factor-alpha system in humans. J Clin Endocrinol Metab 82: 3408-3413, 1997.

9. Hotamisligil GS, Shargill NS and Spiegelman BM: Adipose expression of tumor necrosis factor-alpha: direct role in obesitylinked insulin resistance. Science 259: 87-91, 1993.

10. Khamzina L, Veilleux A, Bergeron S and Marette A: Increased activation of the mammalian target of rapamycin pathway in liver and skeletal muscle of obese rats: possible involvement in obesity-linked insulin resistance. Endocrinology 146: 1473-1481, 2005.

11. Um SH, Frigerio F, Watanabe M, et al: Absence of S6K1 protects against age- and diet-induced obesity while enhancing insulin sensitivity. Nature 431: 200-205, 2004.

12. Lee DF, Kuo HP, Chen CT, et al: IKK beta suppression of TSC1 links inflammation and tumor angiogenesis via the mTOR pathway. Cell 130: 440-455, 2007.

13. Lee DF and Hung MC: All roads lead to mTOR: integrating inflammation and tumor angiogenesis. Cell Cycle 6: 3011-3014, 2007.

14. $\mathrm{Hu} \mathrm{MC}$, Lee DF, Xia W, et al: IKappaB kinase promotes tumorigenesis through inhibition of forkhead FOXO3a. Cell 117: 225-237, 2004.
15. Harmon AW, Paul DS and Patel YM: MEK inhibitors impair insulin-stimulated glucose uptake in 3T3-L1 adipocytes. Am J Physiol Endocrinol Metab 287: E758-E766, 2004.

16. Yuan M, Konstantopoulos N, Lee J, et al: Reversal of obesityand diet-induced insulin resistance with salicylates or targeted disruption of Ikkbeta. Science 293: 1673-1677, 2001.

17. Ou-Yang F, Lan KL, Chen CT, et al: Endostatin-cytosine deaminase fusion protein suppresses tumor growth by targeting neovascular endothelial cells. Cancer Res 66: 378-384, 2006.

18. Hotamisligil GS, Peraldi P, Budavari A, Ellis R, White MF and Spiegelman BM: IRS-1-mediated inhibition of insulin receptor tyrosine kinase activity in TNF-alpha- and obesity-induced insulin resistance. Science 271: 665-668, 1996.

19. Wellen KE and Hotamisligil GS: Inflammation, stress, and diabetes. J Clin Invest 115: 1111-1119, 2005.

20. Garris DR and Garris BL: Cytochemical analysis of pancreatic islet hypercytolipidemia following diabetes $(\mathrm{db} / \mathrm{db})$ and obese (ob/ob) mutation expression: influence of genomic background. Pathobiology 71: 231-240, 2004.

21. Arkan MC, Hevener AL, Greten FR, et al: IKK-beta links inflammation to obesity-induced insulin resistance. Nat Med 11: 191-198, 2005.

22. Cai D, Yuan M, Frantz DF, et al: Local and systemic insulin resistance resulting from hepatic activation of IKK-beta and NF-kappaB. Nat Med 11: 183-190, 2005.

23. Weisberg SP, McCann D, Desai M, Rosenbaum M, Leibel RL and Ferrante AW Jr: Obesity is associated with macrophage accumulation in adipose tissue. J Clin Invest 112: 1796-1808, 2003.

24. Rose DP, Komninou D and Stephenson GD: Obesity, adipocytokines, and insulin resistance in breast cancer. Obes Rev 5: 153-165, 2004. 